\title{
Viral Cell Proliferation
}

National Cancer Institute

\section{Source}

National Cancer Institute. Viral Cell Proliferation. NCI Thesaurus. Code C19107.

A change in the rate of division or growth of cells that have undergone cellular transformation, which is usually induced either by infection with or genomic integration of a transforming virus. 